\title{
Exotic topological types of Majorana zero modes and their universal quantum manipulation
}

\author{
Y. X. Zhao* and Z. D. Wang ${ }^{\dagger}$ \\ Department of Physics and Center of Theoretical and Computational Physics, The University of Hong Kong, \\ Pokfulam Road, Hong Kong, China \\ (Received 14 February 2014; revised manuscript received 15 September 2014; published 30 September 2014)
}

\begin{abstract}
From our general index theorem, which characterizes faithfully the topological intrinsic boundary-bulk correspondence of topological superconductors and insulators, we reveal rigorously that four topologically distinct types of Majorana zero modes can emerge at the ends of superconducting wires of various symmetry classes. More intriguingly, we establish three exotic one-dimensional models that have different types of topological charge of Majorana zero modes and disclose exactly the corresponding topological properties, whose distinct topological essences may be tested experimentally. Moreover, we also address their application in universal quantum manipulation, which is promising for realizing universal topological quantum computation.
\end{abstract}

DOI: 10.1103/PhysRevB.90.115158

PACS number(s): 71.10.Pm, 03.65.Vf, 03.67.Lx

\section{INTRODUCTION}

Recently, the realization of Majorana fermions (MFs) [1,2], which are their own antiparticles and usually obey non-Abelian statistics, has attracted a lot of attention both theoretically [3-13] and experimentally [14-17], because of not only the novel physics but also their potential applications for topological quantum computation [18-24]. In particular, topologically protected Majorana zero modes [25] residing at the ends of one-dimensional topological superconductor (TSC) wires are of great interest, since they are pointlike particles topologically stable against disorders and perturbations preserving the symmetries of a corresponding class. The relevant essence was elucidated by Kitaev, i.e., how a nontrivial bulk topological configuration can lead to unpaired Majorana zeroin modes at the two ends of the superconducting wire [3,4], not only through illustrating a toy model of spinless $p$-wave superconductors, but also, more importantly, by establishing the topology-related theoretical formalism. Inspired by Kitaev's pioneering work, various theoretical proposals and experimental endeavors have been undertaken for realizing and detecting MFs in one-dimensional (1D) materials [5-10,13-17], making it very promising to find MFs in 1D TSCs first. However, these proposals are almost all focused on concrete 1D systems with an implicit assumption that only one topological type of MF, like that in the Kitaev model, may exist even for 1D models with different symmetry classes. Here we reveal that four topologically distinct types of Majorana zero mode can actually emerge at the ends of 1D TSCs, based on a faithful quantitative correspondence between the topological charge of Fermi surfaces on the boundary and the topological number in the bulk [26-30]. In particular, from a general index theorem established by us [30], we construct three exotic 1D models that have different types of topological charge of Majorana zero modes and study rigorously the corresponding topological properties.

Let us first review briefly the bulk-boundary correspondence. We recall that a complete classification was obtained for strong topological insulators (TIs) and TSCs in all eight real Altland-Zirnbauer (AZ) symmetry classes, considering

\footnotetext{
*zhaoyx@hku.hk

†zwang@hku.hk
}

the time-reversal symmetry (TRS), particle-hole symmetry (PHS), or/and chiral symmetry (CS), as illustrated in Table I in a right-to-left manner [26-28,30,31]. Recently, the complete classification of Fermi surfaces (FSs) was also achieved by considering the same set of symmetries [29,30], through generalizing the primary topological charge of Fermi surfaces [32-34] as illustrated in Table I in a left-to-right manner, and the one-to-one relation between the classification of FSs and that of TIs/TSCs has also been revealed rigorously [30]. Moreover, a general index theorem describing quantitatively the topological boundary-bulk correspondence of TIs/TSCs was established and proven [30,35]. It is written as

$$
v_{L / R}(d-1, i)= \pm N(d, i),
$$

where $v_{L / R}$ denotes the total topological charge of FSs (zero modes) on the the left/right boundary of a given bulk TI (or TSC), corresponding to the $+/-$ sign in Eq. (1), and $N$ is the topological number of the bulk, with $d$ and $i$ denoting respectively the bulk dimension and the symmetry class. It is emphasized that the above general index theorem of Eq. (1) also indicates that the type of topological charge of FSs is the same as that of the bulk topological number. Note that for a $\mathbf{Z}$-type TI/TSC, the topological charges of FSs at the left and right boundaries have opposite signs, while for $\mathbf{Z}_{2}$ type, the sign of the topological charge plays no role. For $d=1$, this general index theorem suggests that the models may have Majorana zero modes (FSs) with different topological origins from that of the Kitaev model in class D, making it possible for us to have a deeper insight into all topological types of Majorana zero modes at the ends of TSC wires, with exotic topological properties and phenomena as well as applications being expected. Models in all symmetry classes can be constructed from a generic form in momentum space, $\mathcal{H}=\sum_{i j} f_{i j}(k) \sigma_{i} \otimes \tau_{j}$, where the $f(k)$ 's are even or odd functions determined by the required symmetries and $\sigma$ and $\tau$ are Pauli matrices. We shall construct nontrivial models in the other three symmetry classes below, exploring their topological origins and their properties with comparisons to the Kitaev model in class D.

\section{DIII MODEL}

Let us begin by constructing an intriguing model in class DIII, which has a TRS of negative sign and a PHS of positive 
TABLE I. Classification table of topological types for FSs and TIs/TSCs. The upper part shows the AZ real symmetry classes. $d, p$, and $i$ denote the spatial dimension of a TI/TSC, the codimension of an FS, and the index of symmetry classes, respectively. Four topological types of MF are indicated in the first row of the lower part.

\begin{tabular}{lccccccccc}
\hline \hline $\mathrm{FS}$ & $\mathrm{AI}$ & $\mathrm{BDI}$ & $\mathrm{D}$ & $\mathrm{DIII}$ & $\mathrm{AII}$ & $\mathrm{CII}$ & $\mathrm{C}$ & $\mathrm{CI}$ & $\frac{\mathrm{TI}}{\mathrm{TSC}}$ \\
\hline $\mathrm{T}$ & +1 & +1 & 0 & -1 & -1 & -1 & 0 & +1 & $\mathrm{~T}$ \\
$\mathrm{C}$ & 0 & +1 & +1 & +1 & 0 & -1 & -1 & -1 & $\mathrm{C}$ \\
$\mathrm{S}$ & 0 & 1 & 0 & 1 & 0 & 1 & 0 & 1 & $\mathrm{~S}$ \\
$p \backslash i$ & 1 & 2 & 3 & 4 & 5 & 6 & 7 & 8 & $i / d$ \\
\hline 0 & 0 & $\mathbf{Z}$ & $\mathbf{Z}_{2}^{(1)}$ & $\mathbf{Z}_{2}^{(2)}$ & 0 & $2 \mathbf{Z}$ & 0 & 0 & 1 \\
1 & 0 & 0 & $\mathbf{Z}$ & $\mathbf{Z}_{2}^{(1)}$ & $\mathbf{Z}_{2}^{(2)}$ & 0 & $2 \mathbf{Z}$ & 0 & 2 \\
2 & 0 & 0 & 0 & $\mathbf{Z}$ & $\mathbf{Z}_{2}^{(1)}$ & $\mathbf{Z}_{2}^{(2)}$ & 0 & $2 \mathbf{Z}$ & 3 \\
\hline \hline
\end{tabular}

sign, and thus may have a $\mathbf{Z}_{2}^{(2)}$-type nontrivial topological number, as seen in Table I. According to our general index theorem, the Majorana zero modes at the ends in class DIII should have a $\mathbf{Z}_{2}^{(2)}$-type topological charge that topologically protects these zero modes, in contrast to those of the Kitaev model, which have a $\mathbf{Z}_{2}^{(1)}$-type topological charge, recalling that the Kitaev model is in class D with only a PHS of positive sign. Just as in higher dimensions where the two $\mathbf{Z}_{2}$-type topological charges correspond to different configurations of the Fermi surfaces $[29,30]$, in contrast to the fact that they are not distinguished in earlier works [26-28], it is expected that the Majorana zero modes in the present DIII model are essentially different from those in the Kitaev model. To be explicit, the unique features of our DIII model are listed as follows. (i) Our DIII model in its topological phase has, at each end, two Majorana zero modes of orthogonal spin pairings as the eigenmodes of $\sigma_{x}$, unlike that in the Kitaev model where only one spinless Majorana zero mode resides at each end. (ii) The ground-state degeneracy is 4, twice the ground-state degeneracy of the Kitaev model. (iii) The relatively rich diversity of this DIII model creates more possibilities for quantum manipulation, such as quantum information storage and processing. Most significantly, this DIII model with one Majorana fermion being generated at each end is in a twofold-degenerate space, which may be utilized as a physical qubit for universal quantum manipulation, namely, an arbitrary given state may be created by coupling the system to a weak magnetic field. In contrast, only one quantum gate can be realized with the conventional method using two copies of the Kitaev models through complicated braiding operations $[22,23]$. Furthermore, there exists no difficulty in principle in realizing universal quantum computation with additional braiding operations acting on two copies of our DIII model, while for the Kitaev model this is in principle impossible.

We now introduce our DIII model, whose Hamiltonian reads

$$
\begin{aligned}
H_{\mathrm{DIII}}= & \sum_{j}\left[-w\left(a_{j}^{\dagger} a_{j+1}+a_{j+1}^{\dagger} a_{j}\right)-\mu\left(a_{j}^{\dagger} a_{j}-1\right)\right] \\
& -\Delta \sum_{j}\left(a_{j+1} i \sigma_{1} a_{j}+a_{j}^{\dagger}\left(-i \sigma_{1}\right) a_{j+1}^{\dagger}\right) \\
= & \int d k \Psi_{k}^{\dagger}\left[(-w \cos k-\mu / 2) \mathbf{1} \otimes \tau_{3}\right. \\
& \left.-\Delta \sin k \sigma_{3} \otimes \tau_{1}\right] \Psi_{k},
\end{aligned}
$$

where $w, \mu$, and $\Delta$ are all real, $\sigma_{i}$ and $\tau_{i}$ are Pauli matrices, $\Psi_{k}^{\dagger}=\left[a_{k}^{\dagger}, a_{-k}\left(-i \sigma_{2}\right)\right]$, and the spin index is implicit. From the expression of $\mathcal{H}_{\mathrm{DIII}}(k)$ in the second equality of Eq. (2), it is seen that the model has a PHS with $C=\tau_{1}$ and a TRS with $T=\sigma_{2}$ [36], which means that it belongs to class DIII. The model may be viewed as a spin-triplet Bogoliubov-de Gennes model, which is different from the existing models utilizing spin-orbital couplings to realize nontrivial topology [11,12]. The DIII model may be diagonalized in its spin space under the eigenstates of $\sigma_{x}$, consisting of two Kitaev chains with the signs of order parameters being opposite. However, after bosonization, one chain interacts with a gauge field given by the particle density in the other chain [37]. Notably, a topology-preserved quantum simulation of the present DIII model has very recently been proposed in Ref. [38] using experimentally feasible superconducting quantum circuits, with a special gauge structure being realized by a dispersive dynamic modulation approach. According to Table I, it may have a $\mathbf{Z}_{2}^{(2)}$-type topological number. From the calculation given in Appendix A, the regime of $|w|>|\mu / 2|$ is identified as the topological phase and the corresponding phase diagram is shown in Fig. 2. The nontrivial topological number implies Majorana zero modes of $\mathbf{Z}_{2}^{(2)}$-type topological charge at each end from our general index theorem Eq. (1). So we now examine these Majorana zero modes in detail.

As usual, the Majorana operators can be decomposed as

$$
\begin{aligned}
& \gamma_{j s}^{1}=e^{i \theta / 2} a_{j s}+e^{-i \theta / 2} a_{j s}^{\dagger}, \\
& \gamma_{j s}^{2}=\left(e^{i \theta / 2} a_{j s}-e^{-i \theta / 2} a_{j s}^{\dagger}\right) / i,
\end{aligned}
$$

where $\gamma^{a}$ with $a=1,2$ satisfy $\gamma^{\dagger}=\gamma$, and $s$ is the spin index. We point out that $\theta$ in the above decomposition can be chosen arbitrarily, and is physically indistinguishable in our DIII model, in contrast to the $\theta$ in the Kitaev model which is related to the phase of the Cooper-pairing order-parameter. Since the existence of unpaired Majorana zero modes and their features are topologically intrinsic according to the general index theorem Eq. (1), to see the structure of these zero modes it is sufficient to solve the model exactly at one specific point in the topological phase $[3,4]$. For simplicity, adopting the convention that $\theta=\pi / 2$, let us focus on the case $w=\Delta$ and $\mu=0$ in the topological phase, where our DIII model (2) is reduced to

$$
H_{\mathrm{DIII}}=\sum_{j} i w \gamma_{j,+}^{2} \gamma_{j+1,+}^{1}+\sum_{j}-i w \gamma_{j,-}^{1} \gamma_{j+1,-}^{2},
$$

where the \pm are two eigen spin pairings with respect to $\sigma_{x}$, i.e.,

$$
a_{j,+}=\frac{1}{\sqrt{2}}\left(a_{j, \uparrow}+a_{j, \downarrow}\right), \quad a_{j,-}=\frac{1}{\sqrt{2}}\left(a_{j, \uparrow}-a_{j, \downarrow}\right) .
$$

It is observed that $\gamma_{j, \alpha}^{2}$ and $\gamma_{j+1, \alpha}^{1}$ (with $\alpha= \pm$ ) can pair together to form new electronic operators

$$
\tilde{a}_{j,+}=\frac{1}{2}\left(\gamma_{j,+}^{2}+i \gamma_{j+1,+}^{1}\right), \quad \tilde{a}_{j,-}=\frac{1}{2}\left(\gamma_{j+1,-}^{2}+i \gamma_{j,-}^{1}\right),
$$

to fully diagonalize the Hamiltonian. The pairing pattern of Majorana operators under the open-boundary condition is 


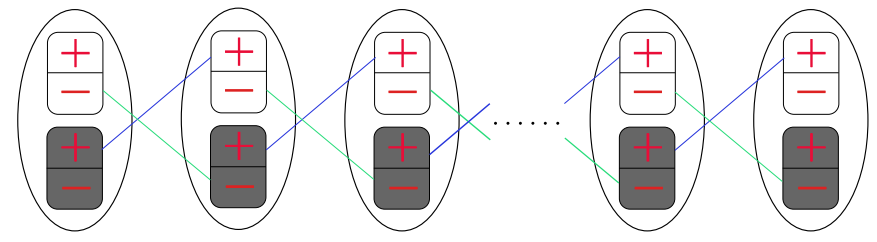

FIG. 1. (Color online) The Majorana repairing of the DIII model.

pictured in Fig. 1 . It is observed that $\gamma_{1,+}^{1}$ and $\gamma_{N_{s},+}^{2}$ (with $N_{s}$ being the total number of sites) are unpaired without entering the Hamiltonian at the two ends, and so are $\gamma_{1,-}^{2}$ and $\gamma_{N_{s},-}^{1}$. In other words, $\gamma_{1,+}^{1}$ and $\gamma_{1,-}^{2}$ are the Majorana zero modes at the left end, while $\gamma_{N_{s},+}^{2}$ and $\gamma_{N_{s},-}^{1}$ are the ones at the right end. To compare with the Kitaev model of $\mathbf{Z}_{2}^{(1)}$ type, recall that only one Majorana operator is unpaired at each end in its topological phase.

It is observed that $\gamma_{1,+}^{1}$ and $\gamma_{1,-}^{2}$ together have nontrivial $\mathbf{Z}_{2}^{(2)}$-type topological charge $v_{L}=1$ at the left end, while $\gamma_{N_{s},+}^{2}$ and $\gamma_{N_{s},-}^{1}$ also have $v_{R}=1$ at the right end. Since the Majorana zero modes at the two ends have the same topological charge, they should be able to be deformed into each other continuously. As $\theta$ in our DIII model can be arbitrarily chosen, it is seen that $\gamma_{j}^{1}$ is transformed to $\gamma_{j}^{2}$ after rotating $\theta$ by $\pi$ in the general Majorana decomposition, confirming that Majorana zero modes at each end are indeed topologically equivalent, in agreement with their $\mathbf{Z}_{2}$ nature. For comparison with the $\mathbf{Z}_{2}^{(1)}$-type Kitaev model, $\theta$ in the present DIII model is an intrinsic freedom such that varying it keeps the Hamiltonian invariant, while its variation in the Kitaev model changes the Hamiltonian explicitly in a topologically equivalent family.

This result, although obtained at a specific point in the topological phase, can be generalized to the general situation according to the concept of topological protection. The Majorana zero mode represented by $\gamma_{1,+}^{1}$, for instance, is replaced by $\Gamma_{+}^{L}$ as a linear combination of Majorana operators with real coefficients and + spin pairing, and the other three Majorana zero modes are replaced by $\Gamma_{-}^{L}, \Gamma_{+}^{R}$, and $\Gamma_{-}^{R}$, respectively. For all of the four combinations, the coefficients decay exponentially away from the ends in the thermodynamic limit $N_{s} \rightarrow \infty$. Because four Majorana operators do not enter the Hamiltonian, the ground state is fourfold degenerate with the basis

$$
\left\{\left|\psi_{1}^{+} \psi_{1}^{-}\right\rangle,\left|\psi_{0}^{+} \psi_{1}^{-}\right\rangle,\left|\psi_{1}^{+} \psi_{0}^{-}\right\rangle,\left|\psi_{0}^{+} \psi_{0}^{-}\right\rangle\right\}
$$

The first has no Majorana fermion, the second and the third have one Majorana fermion at each end, and the last one has two Majorana fermions at each end. Since $-i \Gamma_{\alpha}^{L} \Gamma_{\alpha}^{R}\left|\psi_{0,1}^{\alpha}\right\rangle=$ $\pm\left|\psi_{0,1}^{\alpha}\right\rangle$, it is straightforward to see that the states with no Majorana fermion and with two Majorana fermions at each end have even fermionic parity, i.e., the number of fermions is even, and the two states with one Majorana at each end have odd fermionic parity. Also, for comparison, we recall that the ground state of the Kitaev model with one Majorana fermion at each end has even fermionic parity, while the ground state without Majorana fermions has odd fermionic parity [3].
The ground-state space spanned by $|\bullet\rangle=\left|\psi_{0}^{+} \psi_{1}^{-}\right\rangle$and $|x\rangle=\left|\psi_{1}^{+} \psi_{0}^{-}\right\rangle$with one Majorana fermion at each end has odd parity, distinguishing it from the other ground states. Because of the orthogonal spin pairings for $|\bullet\rangle$ and $|x\rangle$, the degeneracy may be lifted by coupling the spin degree of the DIII model to an external source, and therefore the space can be regarded as a qubit of which any state is achievable. Let us demonstrate this by coupling all electron spins to a very weak external magnetic field compared with the bulk energy gap, i.e., $H^{\prime}=\sum_{j} \mathbf{B} \cdot \sigma_{j} / 2$ as a perturbation. After detailed calculations in the electronic representation in Appendix $\mathrm{C}$, we have the low-energy effective results $\left\langle\bullet\left|\hat{S}_{x}\right| \bullet\right\rangle=1 / 2$, $\left\langle\times\left|\hat{S}_{x}\right| \times\right\rangle=-1 / 2,\left\langle\bullet\left|\hat{S}_{y, z}\right| \bullet\right\rangle=0$, and $\left\langle\times\left|\hat{S}_{y, z}\right| \times\right\rangle=0$ under the thermodynamic limit, with $\hat{\mathbf{S}}=\sum_{j} 1 / 2 \sigma_{j}$. An intuitive picture of this feature is obviously that the MF states are wave packets entering at the ends, with the specific spin orientations being the only degrees that are sensitive to the external magnetic field, irrelevant to the homogeneous bulk state. Thus as the symbols suggest, $\hat{\mathbf{S}}$ may be effectively regarded as a single spin operator acting on the two-level space with $|\bullet\rangle$ and $|x\rangle$ being the two eigenstates of $\hat{S}_{x}$. Thus through a suitable control of $H^{\prime}(t)$ with $H^{\prime}(t \leqslant 0)=H^{\prime}\left(t \geqslant t_{f}\right)=0$, an arbitrary state can be created, leading to a universal single-qubit operation. Notably, spatial fluctuations of the above weak field would have no impact on the decoherence of the ground state. The magnetic field response of DIII models without retaining the fermionic parity was addressed in Refs. [11,12], where Rashba-type spin-orbital coupling was utilized to realize nontrivial topological phases. In Ref. [12], a highly anisotropic response to the magnetic field was studied for a DIII model, which confirms the conclusion in our DIII model.

\section{BDI MODEL}

The Hamiltonian of our BDI model reads

$$
\begin{aligned}
H_{\mathrm{BDI}}= & \sum_{j}\left(-w a_{j}^{\dagger} a_{j+1}+i \Delta a_{j} a_{j+1}\right)+\text { H.c. } \\
& -\mu\left(a_{j}^{\dagger} a_{j}-\frac{1}{2}\right) \\
= & \int d k \Psi_{k}^{\dagger}\left[-\Delta \sin k \tau_{1}-(w \cos k+\mu / 2) \tau_{3}\right] \Psi_{k},
\end{aligned}
$$

where $\Delta$ is still real, $a_{j}$ are spinless, and therefore $\Psi_{k}=$ $\left(a_{k}, a_{-k}^{\dagger}\right)^{T}$. It is noted that $\mathcal{H}_{\mathrm{BDI}}(k)$ in the second equality has TRS with $T=\tau_{3}$ and PHS with $C=\tau_{2}$, corresponding to the class BDI. It is observed that the BDI model may be regarded as a reduced Kitaev model with the phase of the order parameter being fixed; therefore it may be realized by hard-core bosons [38,39]. According to Table I, the formula for the $\mathbf{Z}$-type topological number is used in Appendix A, and the resulted phase diagram is plotted in Fig. 2. In topological phases, i.e., where $|w|>|\mu / 2|$, there exist Majorana zero modes at the two ends, noting that now $N= \pm 1$ are distinguishable, corresponding to different topological phases. The Majorana decompositions adopted are

$$
\gamma_{j}^{1}=e^{i \frac{\theta}{2}} a_{j}+e^{-i \frac{\theta}{2}} a_{j}^{\dagger} \quad \gamma_{j}^{2}=\left(e^{i \frac{\theta}{2}} a_{j}-e^{-i \frac{\theta}{2}} a_{j}^{\dagger}\right) / i .
$$




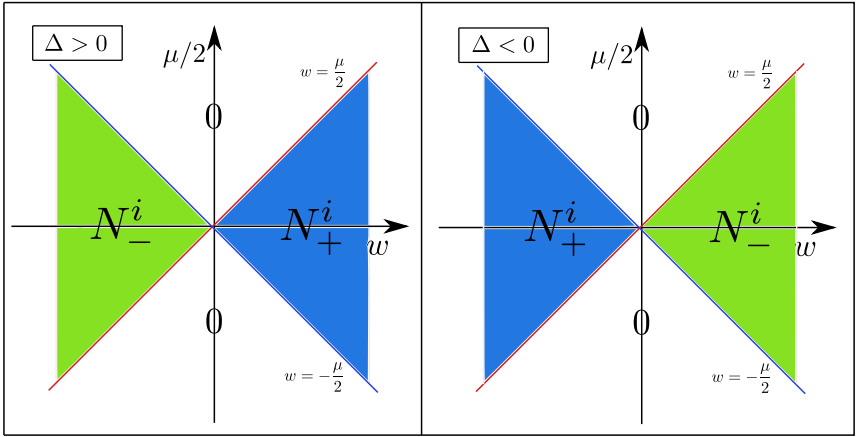

FIG. 2. (Color online) Topological phase diagram. $N_{ \pm}^{i}$ denotes the topological number of each model in the $i$ th class. Specifically, $N_{+}^{D}=N_{-}^{D}=1 \bmod 2, N_{+}^{\mathrm{BDI}}=-N_{-}^{\mathrm{BDI}}=1, N_{+}^{\mathrm{DIII}}=N_{-}^{\mathrm{DIII}}=$ $1 \bmod 2$, and $N_{+}^{\mathrm{CII}}=-N_{-}^{\mathrm{CII}}=2$.

We investigate the Majorana zero modes for $N=+1$, with the representative point in the topological phase being chosen as $w=\Delta>0$ and $\mu=0$, which is exactly solvable. Through fixing $\theta=\pi / 2$ in the Majorana decompositions, we reduce the Hamiltonian as

$$
H_{\mathrm{BDI}}=\sum_{j} i w \gamma_{j}^{2} \gamma_{j+1}^{1},
$$

which can be fully diagonalized by using the repaired electronic operator $\tilde{a}_{j}=\frac{1}{2}\left(\gamma_{j}^{2}+i \gamma_{j+1}^{1}\right)$. It is observed that $\gamma_{1}^{1}$ and $\gamma_{N_{s}}^{2}$ do not enter the Hamiltonian, serving as Majorana zero modes at the two ends. According to the general index theorem (1), the $\mathbf{Z}$-type topological charges $v_{L}\left[\gamma_{1}^{\prime 1}\right]=+1$ and $v_{R}\left[\gamma_{N_{s}}^{\prime 2}\right]=-1$ for the two zero modes can respectively be assigned at the two ends, with the prime indicating that $\theta=\pi / 2$ in the Majorana decompositions.

The simple case of the topological phase with $N=-1$ is that of $w=-\Delta>0$ and $\mu=0$. We can still simplify the Hamiltonian by using the Majorana decompositions, but in this case fixing $\theta=-\pi / 2$. In this phase, similarly $\gamma_{1}^{\prime \prime 1}$ and $\gamma_{N_{s}}^{\prime \prime 2}$ are Majorana zeromodes at the two ends with the double prime denoting that $\theta=-\pi / 2$ is different from that in the phase $N=$ +1 . The general index theorem (1) implies that $v_{L}\left[\gamma_{1}^{\prime \prime 1}\right]=-1$ and $v_{R}\left[\gamma_{N_{s}}^{\prime \prime 2}\right]=1$. It is simple to check that $\gamma_{j}^{\prime \prime 1}=\gamma_{j}^{\prime 2}$ and $\gamma_{j}^{\prime \prime 2}=-\gamma_{j}^{\prime 1}$, which conforms to our general index theorem.

There exists a nontrivial way to realize the topological phase transition through breaking the TRS, an antiunitary symmetry, without closing the gap in the bulk, which is essentially different from ordinary phase transitions breaking a unitary continuous symmetry in Landau's paradigm. We can break the TRS with $T=\tau_{3}$ to make the system in class D through, replacing $i \Delta$ in Eq. (4) by $|\Delta| e^{i \theta}$. Then $\theta= \pm \pi / 2$ correspond to the two topological phases of $N= \pm 1$, respectively, and the two phases can be connected by continuously varying $\theta$ from $\pi / 2$ to $-\pi / 2$ with $|\Delta|$ fixed, which implies intuitively that the two Majorana zero modes residing respectively at the left and right ends are exchanged. The two ways to realize the phase transition are illustrated in Fig. 3. Furthermore, we point out that during the process of varying $\theta$, the system is protected by a nontrivial $\mathbf{Z}_{2}^{(1)}$-type topology, while only when $\theta= \pm \pi / 2$ is the $\mathbf{Z}$-type topological protection recovered, showing that our BDI model is just the Kitaev model whenever

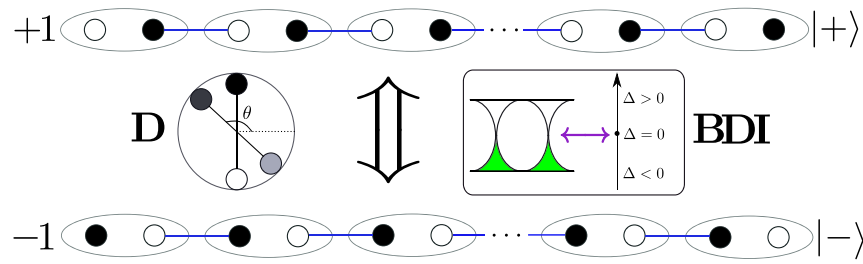

FIG. 3. (Color online) Phase transition of the BDI model.

$\Delta$ is complex [3]. To see the distinction of the two phases clearly, it is noted that the ground states of the two phases under open-boundary condition are orthogonal to each other in the thermodynamic limit as is derived in Appendix B; this is expressed as $\left\langle\psi_{\alpha}, 1 \mid \psi_{\beta},-1\right\rangle=0$ with $\left|\psi_{\alpha}, \pm 1\right\rangle$ being a generic ground state for $N= \pm 1$ [40], which serves as a refinement of the ground-state orthogonality of the Kitaev model.

\section{CII MODEL}

We finally briefly describe our exotic model in class CII, with 2Z-type topological number $N= \pm 2$ in its topological phases, as is calculated in Appendix A. The Hamiltonian reads

$$
\begin{aligned}
H_{\mathrm{CII}}= & \sum_{j}\left(-\Delta a_{j+1}^{\dagger} i \sigma_{2} a_{j}+w a_{j+1}^{\dagger} i \sigma_{2} a_{j}^{\dagger}\right. \\
& \left.-\mu a_{j}^{\dagger} i \sigma_{2} a_{j}^{\dagger}\right)+ \text { H.c. } \\
= & \int d k \Psi_{k}^{\dagger}\left(-\Delta \sin k \sigma_{2} \otimes \tau_{3}\right. \\
& \left.+(w \cos k-\mu) \mathbf{1} \otimes \tau_{1}\right) \Psi_{k},
\end{aligned}
$$

where $\Delta$ and $w$ are still real, and $\Psi_{k}$ is the same as that of the DIII model (2). $\mathcal{H}_{\mathrm{CII}}(k)$ in the second equality has a TRS with $T=\sigma_{2} \otimes \mathbf{1}$ and a PHS with $C=\mathbf{1} \otimes \tau_{2}$. Although the Hamiltonian takes an exotic spin-pairing form, whose phase diagram is also plotted in Fig. 2, it may be simulated using various artificial systems.

Straightforward calculation using the corresponding Z-type formula shows that this model has topological number $N=$ \pm 2 when $|w|>|\mu / 2|$ as in Fig. 2 , consistent with $2 \mathbf{Z}$ in Table I of this paper. To see the form of the topologically protected Majorana zero modes at the two ends, when $w=\Delta>0$ and $\mu=0$ corresponding to $N=2$, we adopt the Majorana decompositions of the DIII model with $\theta=0$, and simplify the CII model (4) as

$$
H_{\mathrm{CII}}=\sum_{j} i w \gamma_{j \downarrow}^{2} \gamma_{j+1 \uparrow}^{1}-\sum_{j} i w \gamma_{j \uparrow}^{2} \gamma_{j+1 \downarrow}^{1},
$$

which may be viewed as two copies of the simplified BDI model. The Hamiltonian can be fully diagonalized by introducing

$$
\tilde{a}_{j}=\frac{1}{2}\left(\gamma_{j \downarrow}^{2}+i \gamma_{j+1 \uparrow}^{1}\right) \quad \tilde{b}_{j}=\frac{1}{2}\left(\gamma_{j \uparrow}^{2}+i \gamma_{j+1 \downarrow}^{1}\right),
$$

which is illustrated in Fig. 4. For the left end, $\gamma_{1 \uparrow}^{1}$ and $\gamma_{1 \downarrow}^{1}$ serve as Majorana zero modes, each having $2 \mathbf{Z}$-type topological charge $\nu_{L}\left[\gamma_{\uparrow, \downarrow}^{1}\right]=1$, and accordingly for the right end $v_{R}\left[\gamma_{\uparrow, \downarrow}^{2}\right]=-1$, which follows the general index theorem of Eq. (1) in this paper. The relevant topological charges 


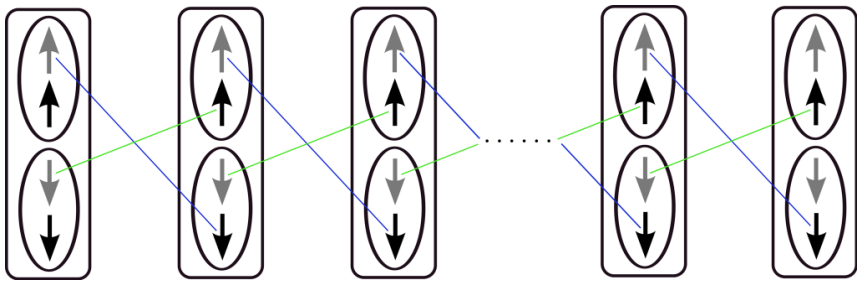

FIG. 4. (Color online) The Majorana repairing of the CII model.

of Majorana zero modes can also be assigned for the other topological phase $N=-2$. Note that the explanation of the CII model in terms of the general index theorem is entirely parallel to that of the BDI model, since they both belong to the $\mathbf{Z}$ group. Although similar to two copies of the BDI model, we wish to indicate that there does not exist a way to connect the two topological phases of the CII model, since breaking either the TRS or PHS makes the topology trivial according to Table I.

\section{SUMMARY}

In summary, all topological types of Majorana zero modes at the ends of one-dimensional topological superconductors have been investigated systematically and rigorously in the framework of the general index theorem. Specifically, aside from Kitaev's model in class D, we have constructed models for the other three classes and solved them exactly at exactly solvable points in their topological phases. Moreover, (i) different topological types of Majorana zero modes have been compared; (ii) the unique properties of each model have been addressed; and (iii) the bulk-boundary correspondence in terms of the general index theorem has explicitly been illustrated for each model. It is anticipated that our exotic results may have applications in universal quantum manipulation, which is promising for realizing universal topological quantum computation.

\section{ACKNOWLEDGMENTS}

We thank J. Teo and S. L. Zhu for helpful discussions. This work was supported by the GRF (Grants No. HKU7045/13P and No. HKU173051/14P), the CRF (Grant No. HKU8/11G) of Hong Kong, and the URC fund of HKU.

\section{APPENDIX A: TOPOLOGICAL NUMBERS}

The DIII model is of the $\mathbf{Z}_{2}^{(2)}$ topological type, and therefore a two-parameter symmetry-preserving extension of $\mathcal{H}_{\text {DIII }}$ is made below to calculate its topological number [29,30]:

$$
\begin{aligned}
\mathcal{H}_{\text {DIII }}(k, \theta, \phi)= & {\left[\mathcal{H}(k) \cos \theta+\sin \theta \mathbf{1} \otimes \tau_{2}\right] \cos \phi } \\
& +\sin \phi \sigma_{1} \otimes \tau_{1} \\
= & (w \cos k+\mu) \cos \theta \cos \phi \mathbf{1} \otimes \tau_{3} \\
& +\sin k \cos \theta \cos \phi \sigma_{3} \otimes \tau_{1} \\
& +\sin \theta \cos \phi \mathbf{1} \otimes \tau_{2}+\sin \phi \sigma_{1} \otimes \tau_{1},
\end{aligned}
$$

where the extended parameters $\theta$ and $\phi$ both range from $-\pi / 2$ to $\pi / 2$. It can be checked that both TRS and PHS are preserved, if we regard $\theta$ and $\phi$ as wave vectors. In the calculation, the following formula has been used:

$$
\begin{aligned}
N_{\text {DIII }}= & \frac{1}{48 \pi^{2}} \int_{0}^{2 \pi} d k \int_{-\pi / 2}^{\pi / 2} d \theta \int_{-\pi / 2}^{\pi / 2} d \phi \epsilon^{\mu \nu \lambda} \\
& \times \operatorname{tr}\left[\sigma_{2} \otimes \tau_{1} \mathcal{H}^{-1} \partial_{\mu} \mathcal{H} \mathcal{H}^{-1} \partial_{\nu} \mathcal{H} \mathcal{H}^{-1} \partial_{\lambda} \mathcal{H}\right] .
\end{aligned}
$$

The BDI model and the CII model are both of $\mathbf{Z}$ type, and therefore their corresponding formulas are [30]

$$
N_{\mathrm{BDI}}=\frac{1}{4 \pi i} \int_{-\pi}^{\pi} d k \operatorname{tr}\left[\tau_{2} \mathcal{H}^{-1}(k) \partial_{k} \mathcal{H}(k)\right]
$$

and

$$
N_{\mathrm{CII}}=\frac{1}{4 \pi i} \int_{-\pi}^{\pi} d k \operatorname{tr}\left[\sigma_{2} \otimes \tau_{2} \mathcal{H}^{-1}(k) \partial_{k} \mathcal{H}(k)\right],
$$

with respect to their chiral symmetries. The results obtained from the above three formulas are illustrated in the phase diagram Fig. 2 in the main text.

\section{APPENDIX B: ORTHOGONALITY OF GROUND STATES OF THE BDI MODEL}

We first solve the ground states of the BDI model exactly in the electronic representation when $w=|\Delta|>0$ and $\mu$ for a generic $\theta$ and then compare the ground states of $\theta=\pi / 2$ and $\theta=-\pi / 2$. The explicit expressions for the two degenerate ground states are

$$
\left|\psi_{0}\right\rangle=\sum_{n=0}^{[N / 2]} \sum_{s_{1}<\cdots<s_{2 n}} e^{-i n \theta} a_{s_{1}}^{\dagger} a_{s_{2}}^{\dagger} \cdots a_{s_{2 n}}^{\dagger}|0\rangle
$$

and

$$
\left|\psi_{1}\right\rangle=\sum_{n=0}^{[(N-1) / 2]} \sum_{s_{1}<\cdots<s_{2 n+1}} e^{-i(n-1) \theta} a_{s_{1}}^{\dagger} a_{s_{2}}^{\dagger} \cdots a_{s_{2 n+1}}^{\dagger}|0\rangle,
$$

where $[r]$ is the integer part of the real number $r,\left|\psi_{0}\right\rangle$ with even parity has a pair of MFs at the two ends, and $\left|\psi_{1}\right\rangle$ with odd parity has no MFs. The inner products of the ground states for $N= \pm 1$ are computed as

$$
\left\langle\psi_{0}, 1 \mid \psi_{0},-1\right\rangle=\frac{C_{N}^{0}-C_{N}^{2}+C_{N}^{4}-\cdots}{C_{N}^{0}+C_{N}^{2}+C_{N}^{4}+\cdots} \rightarrow 0
$$

and

$$
\left\langle\psi_{1}, 1 \mid \psi_{1},-1\right\rangle=\frac{C_{N}^{1}-C_{N}^{3}+C_{N}^{5}-\cdots}{C_{N}^{1}+C_{N}^{3}+C_{N}^{5}+\cdots} \rightarrow 0,
$$

where $\rightarrow$ denotes the thermodynamic limit $N \rightarrow \infty$, and $C_{N}^{n}$ denotes the combination number $N ! /(N-n) ! n !$. It is obvious that $\left\langle\psi_{1} \mid \psi_{0}\right\rangle=0$.

\section{APPENDIX C: RESPONSE OF THE DIII MODEL TO AN EXTERNAL MAGNETIC FIELD}

Referring to the above expressions for $\left|\psi_{0}\right\rangle$ and $\left|\psi_{1}\right\rangle$, the explicit expressions of the four degenerate ground states of the DIII model can also be constructed. Thus every element of the perturbation matrix $\hat{\mathbf{S}}$ can be computed directly. Here we 
present only the most important steps:

$$
\begin{aligned}
\delta E / B & =\left\langle\psi_{0}^{-} \psi_{1}^{+}\left|\sum_{j} \sigma_{j}^{1}\right| \psi_{0}^{-} \psi_{1}^{+}\right\rangle-\left\langle\psi_{0}^{+} \psi_{1}^{-}\left|\sum_{j} \sigma_{j}^{1}\right| \psi_{0}^{+} \psi_{1}^{+}\right\rangle \\
& =\frac{1}{\sum_{\{m=0, n=0\}}^{\{[(L-1) / 2],[L / 2]\}} C_{L}^{2 m+1} C_{L}^{2 n}}\left[\sum_{m}^{[L / 2]} \sum_{n}^{[(L-1) / 2]}(-2 m+2 n+1) C_{L}^{2 m} C_{L}^{2 n+1}-\sum_{n}^{[L / 2][(L-1) / 2]} \sum_{m}^{[L]}(-2 m-1+2 n) C_{L}^{2 m+1} C_{L}^{2 n}\right] \\
& =2+\frac{4 \sum_{m}^{[L / 2]} \sum_{n}^{[(L-1) / 2]}(n-m) C_{L}^{2 m} C_{L}^{2 n+1}}{\sum_{\{m=0, n=0\}}^{\{[(L-1) / 2],[L / 2]\}} C_{L}^{2 m+1} C_{L}^{2 n}} .
\end{aligned}
$$

In the thermodynamic limit, the second term in the last equality vanishes, and thus we have $\delta E / B=2$. This justifies $\left\langle\bullet\left|\hat{S}_{x}\right| \bullet\right\rangle=$ $1 / 2$ and $\left\langle\times\left|\hat{S}_{x}\right| \times\right\rangle=-1 / 2$, and the other matrix elements can be computed similarly.

[1] E. Majorana, Nuovo Cimento 14, 171 (1937).

[2] F. Wilczek, Nat. Phys. 5, 614 (2009).

[3] A. Y. Kitaev, Phys. Usp. 44, 131 (2001).

[4] A. Y. Kitaev, Ann. Phys. (NY) 321, 2 (2006).

[5] R. M. Lutchyn, J. D. Sau, and S. Das Sarma, Phys. Rev. Lett. 105, 077001 (2010).

[6] Y. Oreg, G. Refael, and F. von Oppen, Phys. Rev. Lett. 105, 177002 (2010).

[7] L. Jiang, T. Kitagawa, J. Alicea, A. R. Akhmerov, D. Pekker, G. Refael, J. I. Cirac, E. Demler, M. D. Lukin, and P. Zoller, Phys. Rev. Lett. 106, 220402 (2011).

[8] C. L. M. Wong and K. T. Law, Phys. Rev. B 86, 184516 (2012).

[9] S. Nakosai, J. C. Budich, Y. Tanaka, B. Trauzettel, and N. Nagaosa, Phys. Rev. Lett 110, 117002 (2013).

[10] A. Keselman, L. Fu, A. Stern, and E. Berg, Phys. Rev. Lett. 111, 116402 (2013).

[11] F. Zhang, C. L. Kane, and E. J. Mele, Phys. Rev. Lett. 111, 056402 (2013); 111, 056403 (2013).

[12] E. Dumitrescu, J. D. Sau, and S. Tewari, arXiv:1310.7938.

[13] J. J. He, J. Wu, T.-P. Choy, X.-J. Liu, Y. Tanaka, and K. T. Law, Nat. Commun. 5, 3232 (2014).

[14] V. Mourik, K. Zuo, S. M. Frolov, S. R. Plissard, E. P. A. M. Bakkers, and L. P. Kouwenhoven, Science 336, 1003 (2012).

[15] M. T. Deng, C. L. Yu, G. Y. Huang, M. Larsson, P. Caroff, and H. Q. Xu, Nano Lett. 12, 6414 (2012).

[16] A. Das, Y. Ronen, Y. Most, Y. Oreg, M. Heiblum, and H. Shtrikman, Nat. Phys. 8, 887 (2012).

[17] L. P. Rokhinson, X. Liu, and J. K. Furdyna, Nat. Phys. 8, 795 (2012).

[18] F. Hassler, A. R. Akhmerov, C. Y. Hou, and C. W. J. Beenakker, New J. Phys. 12, 125002 (2010).

[19] F. Hassler, A. R. Akhmerov, and C. W. J. Beenakker, New J. Phys. 13, 095004 (2011).

[20] K. Flensberg, Phys. Rev. Lett. 106, 090503 (2011).

[21] P. Bonderson and R. M. Lutchyn, Phys. Rev. Lett. 106, 130505 (2011).

[22] J. Alicea, Y. Oreg, G. Refael, F. von Oppen, and M. P. A. Fisher, Nat. Phys. 7, 412 (2011).

[23] J. Alicea, Rep. Prog. Phys. 75, 076501 (2012).

[24] C. Nayak, S. H. Simon, A. Stern, M. Freedman, and S. D. Sarma, Rev. Mod. Phys. 80, 1083 (2008).

[25] Majorana zero modes refer to real operators carrying no energy and Majorana fermions refer to eigenstates of these operators in this paper.
[26] A. Y. Kitaev, in Advances in Theoretical Physics: Landau Memorial Conference, edited by V. Lebedev and M. Feigelman, AIP Conf. Proc. No. 1134 (AIP, New York, 2009), p. 22.

[27] A. P. Schnyder, S. Ryu, A. Furusaki, and A. W. W. Ludwig, Phys. Rev. B 78, 195125 (2008); in Advances in Theoretical Physics: Landau Memorial Conference (Ref. [26]), p. 10; S. Ryu, A. P. Schnyder, A. Furusaki, and A. W. W. Ludwig, New J. Phys. 12, 065010 (2010).

[28] J. C. Y. Teo and C. L. Kane, Phys. Rev. B 82, 115120 (2010).

[29] Y. X. Zhao and Z. D. Wang, Phys. Rev. Lett. 110, 240404 (2013).

[30] Y. X. Zhao and Z. D. Wang, Phys. Rev. B 89, 075111 (2014).

[31] A. Altland and M. R. Zirnbauer, Phys. Rev. B 55, 1142 (1997).

[32] G. E. Volovik, The Universe in a Helium Droplet (Clarendon, Oxford, 2003).

[33] G. E. Volovik, Analogue Gravity Phenomenology, Lecture Notes in Physics Vol. 870 (Springer, Berlin, 2013), pp. 343-383.

[34] P. Hořava, Phys. Rev. Lett. 95, 016405 (2005).

[35] Strictly speaking, the index theorem for 1D TIs/TSCs is not explicitly characterized in Ref. [30], since topological charges for pointlike gapless modes are not formulated in Ref. [29], although it seems to be indicated by the eightfold periodicity addressed there. Thus the present work actually completes the index theorem by explicitly revealing its meaning in $1 \mathrm{D}$ TIs/TSCs.

[36] In our convention, the antiunitary operators of TRS and THS are respectively represented as $\hat{T}=T \hat{\kappa}$ and $\hat{C}=C \hat{\kappa}$, where $T$ and $C$ are linear unitary operators, and $\hat{\kappa}$ denotes the basic antiunitary operator of complex conjugation.

[37] The DIII model may be decomposed as $H_{\text {DIII }}=H_{+}+H_{-}$, with $\quad H_{+}=\sum_{j}\left(w a_{j,+}^{\dagger} a_{j+1,+}+\Delta a_{j+1,+} a_{j,+}+\right.$ H.c. $)+$ $\mu\left(a_{j,+}^{\dagger} a_{j,+}-\frac{1}{2}\right)$ and $H_{-}=\sum_{j}\left(w a_{j,-}^{\dagger} a_{j+1,-}-\Delta a_{j+1,-} a_{j,-}+\right.$ H.c. $)+\mu\left(a_{j,-}^{\dagger} a_{j,-}-\frac{1}{2}\right)$, which may be bosonized as $H_{+}=\sum_{j}\left(w e^{i \pi n_{j,-}} b_{j,+}^{\dagger} b_{j+1,+}+\Delta e^{i \pi n_{j,-}} b_{j+1,+} b_{j,+}+\right.$ H.c. $)+$ $\mu\left(b_{j,+}^{\dagger} b_{j,+}-\frac{1}{2}\right) \quad$ and $\quad H_{-}=\sum_{j}\left(w e^{i \pi n_{j+1,+}} b_{j,-}^{\dagger} b_{j+1,-}-\right.$ $\Delta e^{i \pi n_{j+1,+}} b_{j+1,-} b_{j,-}+$ H.c. $)+\mu\left(b_{j,-}^{\dagger} b_{j,-}-\frac{1}{2}\right)$.

[38] Y. Hu, Y. X. Zhao, Z.-Y. Xue, and Z. D. Wang, arXiv:1407.6230.

[39] C.-E. Bardyn and A. İmamoğlu, Phys. Rev. Lett. 109, 253606 (2012).

[40] In principle, we can construct a BDI model with a large topological number $N$, and because it is of $\mathbf{Z}$ type, there are $N$ unpaired Majorana operators at each end without entering the Hamiltonian, corresponding to $2^{N}$-fold ground-state degeneracy. These exotic features provide more possibilities to store and process quantum information, and might be utilized to realize topological quantum computing. 